\title{
Neurological Diseases Associated to Immune Checkpoint Inhibitors
}

Felipe Fanine de Souza ${ }^{*}$, Julia Petry Trevisani ${ }^{1}$, Letícia Caroline Breis ${ }^{1}$, Luís Gustavo Marcelino Sizenando ${ }^{1}$, Marco Antônio Machado Schlindwein ${ }^{1}$, Paola Herreira Silva ${ }^{2}$, Marcus Vinicius Magno Gonçalves ${ }^{3}$

${ }^{1}$ Medical students at University of the Region of Joinville - UNIVILLE - Joinville, Brazil-SC

${ }^{2}$ Medical student at University Positivo - UP - Curitiba, Brazil-PR

${ }^{3}$ Medical Doctor, PhD and Professor of Neurology at University of the Region of Joinville - UNIVILLE - Joinville, Brazil-SC

*Corresponding Author: Felipe Fanine de Souza, Department of Medicine - University of the Region of Joinville. Paulo Malschitzki, 10 -

Zona Industrial Norte, 89201-972, Joinville, Santa Catarina, Brazil

Received date: April 06, 2021; Accepted date: May 07, 2021; Published date: May 13, 2021

Citation: Felipe Fanine de Souza, Julia P. Trevisani., Letícia C. Breis., Marcelino Sizenando LG., Machado Schlindwein MA., (2021) Neurological

Diseases Associated to Immune Checkpoint Inhibitors. J. Neuroscience and Neurological Surgery. 9(1); DOI:10.31579/2578-8868/177

Copyright: () 2021 Felipe Fanine de Souza, This is an open-access article distributed under the terms of The Creative Commons Attribution License, which permits unrestricted use, distribution, and reproduction in any medium, provided the original author and source are credited

\begin{abstract}
:
The treatment for cancer has been more widespread and new therapies appear as alternatives in the area to contain the advance of the tumor, having with the immune mechanisms one of the main sources of research and study for a possible advance in the treatment. Checkpoint inhibitors (ICI) are monoclonal therapy, which act by blocking the PD-1, PD-L1 and CTLA-4 molecules, responsible for immune control. However, among the effects caused by therapy, the use of medications is associated with neurological diseases reported as an adverse effect. Neurological complications can affect both the central and the peripheral nervous system, reaching a variety of regions and being related to effects in several diseases. In clinical practice, the report in question shows how the adverse effects of using these therapies work, collaborating with evidence on the use or not of it. This bibliographic review, which used the PUBMED database with the words "antibodies", "monoclonal", "immune control", "checkpoints inhibitors", brings the main neurological diseases associated with therapy, as well as the incidence, symptoms and treatment.

Methodology: The present review used as a means of obtaining information the PUBMED platform, in which it was looking for articles using the words "" antibodies "," monoclonal "," immune control "," checkpoints inhibitors ", in addition to fulfilling the year criteria between 2010 and 2020. The language and countries in which the data were obtained were not selected, so information from articles published in several countries was used.

Key words: immune checkpoints inhibitors, monoclonal antibodies; neurological diseases; peripheral neuropathy; immune control
\end{abstract}

\section{Introduction}

Immune checkpoint inhibitors (ICI) represent a new form of cancer treatment approved by a variety of cancers like melanoma and lung cancer. They are monoclonal antibodies that act blocking three molecules with other targets being studied [1,2]. The cytotoxic $\mathrm{T}$ lymphocytes associated antigen 4 (CTL4) acts downregulating $\mathrm{T}$ lymphocytes activation by interaction with B7.2 expressed in antigen presenting cell (APC) and regulatory T cell (Treg) [1], while the programmed cell death protein 1 (PD-1) and its ligand (PD-L1), acts limiting T cell activation in peripheral tissues in contrast to CTL4 [1].

This dysregulation of the immune system that brings immune response against cancer cells cause as well important immune related adverse events (irAEs) like pneumonitis, myocarditis, endocrinopathies and neurological events $[3,4]$. Importantly, the association of ICIs is related with much more high-grade adverse events than monotreatments [5].

Neurological adverse events were shown in a systematic review to occur in $3.8 \%$ of the patients exposed to anti-CTL4 treatment, $6.1 \%$ with PD-1 and $12 \%$ in the combination of the two. Most of those were low grade AE like headache $55 \%$. High grade AE on the other hand had a prevalence lower than $1 \%$ [6]. These results mostly from the clinical trials are similar with observational and pharmacovigilance data. In a recent single center study the prevalence of severe nAE was $0.95 \%$ [7], and in a Japanese pharmacovigilance study the prevalence of nAE was 7.67\% [8].

Although neurological AEs are not the most frequent AEs associated with ICI. When we observed the fatal adverse events. Neurological AE together with cardiac $\mathrm{AE}$ represents half the cause of fatal AE in Wang and colleagues' retrospective analyses and $15 \%$ of the fatal $\mathrm{AE}$ from a global pharmacovigilance data [9]. Another important feature is that myocarditis that has the highest fatality rate among all adverse events, frequently co-occur with myositis (25\%-32\%) [10,11]. and Myastenia gravis (MG) $25 \%$ [12].

The use of autoimmune therapies has been widespread in several areas of oncology and, in this context, the study involving these therapies must be understood together in order to obtain the best success and the lowest number of complications for the patient. In this narrative review, we will provide updated information on $\mathrm{nAE}$ related to ICI therapy, its treatment and prognosis, corroborating the analysis of how therapies can influence the patient's context. 
Although not so common, the adverse effects caused by immune therapies in the treatment of cancer are essential for understanding the use or not of this method. Several literatures were used to obtain information, having no restrictions regarding the search for information. Still, future research is needed to understand how the mechanisms occur and how to modify the current parameter of the neurological effects that this therapy acts on. The review in question aimed to elucidate the main neurological side effects caused and, for that, it was based on the following questions: "how does therapy with immune checkpoints inhibitors work?", "What type of cancer most often uses therapy? "," what are the main neurological adverse effects? "," what are the main symptoms, incidence, treatment and prognosis of these adverse effects?".

\section{Peripheral Nervous System}

\subsection{Peripheral neuropathy}

Neuropathies associated with ICI have an estimated incidence of $1 \%$ [13]. Other studies showed an incidence rate of $0.7 \%$ [14]. and $1.28 \%$ [8] when relating neuropathy and immunotherapy. They may vary in severity from typical immune-mediated neuropathy as Guillain-Barré syndrome (GBS) and chronic inflammatory demyelinating polyneuropathy (CIDP) to the small-fiber sensory type (most commonly seen with chemo-therapies) [15-19]. Complications involving the peripheral nervous system (PNS) have been reported in patients treated with anti-PD-1/PD-L1 agents, anti -CTLA4 or using both of them combined. They have consisted in mild to moderate peripheral neuropathies $[6,20,21]$.

In the study by Dubey et al (2014), in which 19 patients with neuropathies relate $\mathrm{AE}$ were identified, the phenotypes associated with ICIs. Were, more prevalently, cranial neuropathy with or without meningitis (7) and polyradiculoneuropathies (6). In addition, it was also noted that the mean time to the start of immunotherapy therapy and the onset of symptoms of neuropathy was 9 weeks. The same study also carried out an analysis comparing ICI-related neuropathy with neuropathies associated with cytotoxic chemotherapies and it was possible to note that melanoma was the type most commonly associated with ICI-associated neuropathies. When seen in relation to neuropathies associated with cytotoxic chemotherapy, adenocarcinoma was the most incident. In addition, hospitalization was more noticeable in patients with neuropathies associated with ICI, to carry out the treatment of adverse events they had. However, in these neuropathies, a better clinical response was also seen when ICI therapy was discontinued or corticosteroids were administered [14].

Although some severe peripheral neuropathies improve significantly with ICI discontinuation, it has been observed a long-term persistence of painful sensory neuropathy even without the medication [14]. In mild cases, there is no need for discontinuation of immune checkpoint inhibitor therapy or initiation of immune modulating treatment such as corticosteroids [20]. Besides the mild peripheral neuropathies, there have been described more widespread cases of inflammations such as meningoradiculitis or meningoradiculonevritis in patients treated with ICIs, mostly with Ipilimumab [22,23]. Recently studies showed that ICI induced peripheral neuropathies can focally or diffusely affect the sensory peripheral roots or motor or limb and can appear as axonal or demyelinating neuropathies [20,21]. Facial weakness and extraocular movement impairment may occur too [14]. Immune related neuropathies can occur after starting ICI therapy and can still be persistent and continue to be manifest even after stopping the immune therapy [24].
The variety of clinical forms involved in neuropathy associated with ICI deserves a review on its own. An interesting association is described by Alhammad et al. (2017): 2 rare cases of brachial plexus neuropathy were observed during treatment with ICI. The 2 cases started after the ninth monoclonal infusion and triggered sudden onset severe pain, in addition to paresis and paresthesia in the hand and upper limb, a clinical presentation compatible with neuralgic amyotrophy [25].

Finally, peripheral neuropathies may manifestate in very different ways raging from Guillain Barre Syndrome (GBS) to mononeuropathy of a single cranial nerve. While usually reversible, there are persistent cases that left long term sequelae. It is highly recommended Neuro-imaging and neurology involvement in this matter [26].

\subsection{Guillain-Barré Syndrome (GBS)}

GBS is a group of autoimmune disorders manifested by acute polyradiculoneuropathy, and it is the most common cause of acute flaccid paralysis [27]. It is estimated that 0.1 to $0.2 \%$ of patients receiving ICI develop acute demyelinating polyneuropathy which resembles GBS ${ }^{16}$. The disease presents in a progressive and symmetrical pattern with ascending sensory and motor dysfunction (paresthesia, muscle weakness, paralysis and sensory loss), autonomic neuropathy and areflexia [16,2831]. Ultimately, GBS triggered by ICI is generally similar to GBS not associated with ICI in terms of presentation and clinical course [32]. Also similarly to GBS not associated with ICI, most cases can be classified as acute inflammatory demyelinating polyneuropathy (AIDP), although rare variants of the syndrome, such as Miller-Fisher syndrome, have also been reported. [33].

Another interesting information is that patients with melanoma may have a higher risk of ICI associated demyelinating polyneuropathy due to shared epitopes in both melanocytes and Schwann cells [34,35].

Corticosteroids are the first-line treatment for GBS caused by ICI [16]. different from idiopathic GBS, in which corticosteroids treatment do not result in significant differences when compared to control groups [36]. This probably happens due to different etiopathogenesis involved in these two situations. Additionally, IVIG or plasmapheresis may be used in cases of poor clinical improvement [16].

\subsection{Chronic Inflammatory demyelinating polyneuropathy}

Chronic inflammatory demyelinating polyneuropathy (CIDP) may occur similarly to GBS in an acute manner in early stages, although can be distinguished by the response to corticosteroids therapy and the time course. The rapid begging of the symptoms suggesting the acute demyelinating polyneuropathy has been described in a lot of cases that were finally diagnosed as CIDP induced by immune checkpoint inhibitors therapy following decompensation weeks after initial improvement $[34,37]$. CIDP is typically related to a slow disease course with time to at least eight weeks. Symptoms may continue progressing or it might occur in a remitting course due to segmental demyelination and remyelination. Changes associated involving neurons may happen either. The mechanisms involved in this process are both humoral and cell mediated [35].

\subsection{Myositis}

Myositis are inflammatory myopathies that induce muscle inflammation associated that could have an extra muscular manifestation associated including cartilage, lung and skin manifestations [38]. Inflammatory myopathies can be classified into polymyositis, dermatomyositis, immune-mediated necrotizing myopathy (IMNM), sporadic inclusion- 
body myositis, and overlap myositis [38,39]. A Meta-analysis study by Psimara et al. (2018) reported the major complications in the peripheral nervous system to ICIs as an AE from the therapy, among complications myositis was the most commonly neurological AE of ICI reported. Patients who are treated with anti-PD-1 aAb like nivolumab have a $1 \%$ chance of being affected by ICI-induced myositis [40].

In the study by Moreira et al. (2019), of the 38 cases of patients who had metastatic cancer and had neuromuscular adverse effects due to therapy using ICI, 19 cases were reported to have myositis, which was the most common AE. Among the 38 cases, 22 were using pembrolizumab, 5 were nivolumab, 2 were ipilimumab and 9 were using combination. In addition, as the symptoms seen in the 38 patients with $\mathrm{AE}$, proximal muscle weakness of the limbs and myalgia were the most frequently seen, with 12 and 16 cases, respectively. It was also analyzed for the presence of autoantibodies associated with myositis in 24 patients, with negative results in $67 \%$ of cases and an interesting fact found in this study is that $32 \%$ of these myositis patients had complications due to myocarditis in combination [10].

Also, the disease present high levels of creatinine kinase and EMG with myopathic pattern, and other less often symptoms as dyspnea, fever, fatigability, chest pain and dysphonia ${ }^{41}$. Other studies have reported that patients who developed myositis can also developed overlapping MG, and presented fluctuation weakness in ocular and bulbar muscles $[42,43]$. Cases of Myositis caused by nivolumab induced use to improve after drug withdrawal and administration of corticosteroid (usually prednisone or prednisolone) with or without immunosuppressive therapy [44]. Prednisone treatment consists in $0,5-1 \mathrm{mg} / \mathrm{kg}$, for patients unresponsive or partially responsive to corticosteroids it may be necessary plasmapheresis or high dose of IVIg administration [42,45-47].

\subsection{Myasthenia gravis}

Myasthenia gravis (MG) as a complication of ICI therapy, which can be seen as an aggravation of the syndrome already possessed or as a new case [34] In a study citing nivolumab, the incidence of MG caused by an adverse effect of ICI therapy was $0.12 \%$, occurring in 12 patients among 9,869 individuals with this therapy $[48,49]$. In the study of Sato et al. (2019), MG associated with ICI was $1.16 \%$ in 7,604 patients analyzed, when compared to the percentage of $0.03 \%$ without using ICI, in 383 patients analyzed [8]. Antibodies to the acetylcholine receptor (AChR) are identified in approximately $85 \%$ of patients with generalized myasthenia gravis and when myasthenia is considered an adverse effect of the use of ICI, positive results for the antibody are found in $66 \%$ of cases [50,51]. When AChR antibodies are detected, the results found are, in most cases, much lower when compared to those found in naive patients with ICI [12,52].

In a Japanese study conducted with nivolumab monotherapy in 9869 cancer patients, there were 12 cases of MG, which started in the initial phase of treatment and evolved rapidly. Markedly high CK levels were obtained in 10 of the 12 patients already diagnosed with MG, in which a mean serum CK of 4799 IU/L was obtained, being a high level that preceded clinical symptoms with MG related to nivolumab, which were also associated with worse prognosis [12]. In addition, it was also noted that of the 12 cases of nivoMG, 10 patients were positive for AChR and that there were 4 cases of myositis, 3 cases of myocarditis and 1 had an association of the myositis and myocarditis, together with the presence of MG. Although these 2 complications mentioned are uncommon events, these disorders can develop simultaneously in patients with MG related to nivolumab [12]. Another study by Sato et al. shows the presence of overlap between myositis and MG in $20 \%$ of patients with MG associated with ICI [8].

In the study carried out by Suzuki S et al. (2017), which makes a comparison of the clinical characteristics between patients who have MG related to nivolumab and idiopathic MG. Dyspnea and limb muscle weakness in patients using ICI therapy were the most common presentations $(67 \%)$. In idiopathic disease, diplopia (75\%) and ptosis (85\%) appear as the 2 most common symptoms caused [12,51]. Symptoms usually progress rapidly with frequent decompensation of the myasthenic crisis, which requires respiratory support. Almost all patients reported with MG related to ICI therapy required hospitalization, with 40$50 \%$ of these patients requiring mechanical ventilation. Which can be associated with patients with high CK levels, according to a study by Safa et al. (2019) [51]. In a retrospective cohort of 65 patients diagnosed with MG induced by ICI, the mean time from the onset of symptoms to respiratory failure and intubation was only 7 days [12]. This is notably distinct from myasthenia gravis not associated with ICI, which has as estimated risk of 15 to $20 \%$ over the life of myasthenic crisis, and in which just one fifth of patients have a myasthenic crisis at the time the diagnosis was made $[53,54]$. The evolution time from the onset of symptoms to the most severe symptoms in patients with MG related to ICI is from 1 to 60 days. On the other hand, in patients with idiopathic MG, the evolution time of the symptoms is approximately 2-3 years [51].

According to Suzuki et al. (2017), treatment using immunosuppressive therapy was effective in patients who had MG related to nivolumab, in which patients with mild symptoms responded to oral corticosteroids and the symptoms were relieved within weeks. However, more severe patients experienced a more delayed and gradual improvement, with 4 to 8 weeks [12]. Regarding the results of treatment and prognosis of the disease, in the study carried out by Safa et al. (2019), the symptoms of AE were completely resolved in $19 \%$ of patients, improved in $55 \%$ and worsened in $26 \% .63 \%$ of the 38 patients who received first-line corticosteroid therapy improved their symptoms, while in the rest of the patients there was an evolution to respiratory failure. When IVIG or PLEX was used as the primary treatment, $95 \%$ of patients showed improvement in symptoms. In addition, death was reported in $37 \%$ of the patients, of which $23 \%$ were due possible complications from MG, after approximately 6 weeks after the initial MG symptoms, and the remaining deaths were due cancer progression, other comorbidities or were not identified [51].

\section{Central nervous system \\ 3.1. Encephalitis}

Encephalitis is an acute inflammation of the brain manifested by neurological symptoms such as headache, confusion, behavior changes and sensorimotor dysfunctions [20,55]. ICIs can cause autoimmune encephalitis with an estimated incidence between $0,1 \%$ and $0,25 \%$, and this is especially related to the combined therapy of anti-CTLA4 (ipilimumab) and anti-PD1 (nivolumab) [55,56].

A retrospective study by Larkin et al. (2017) investigated immune-related $\mathrm{AE}$ in patients with advanced melanoma treated with nivolumab with or without ipilimumab. 3763 patients were included, and 35 patients of those $(0.93 \%)$ had severe neurological AE. Encephalitis was the second most common neurological AE, happening in 6 patients, only behind neuropathy. Also, the median time of encephalitis onset was 51.5 days 
[20]. Additionally, limbic encephalitis has also been reported while using pembrolizumab [57].

Vogrig et al. (2019) reported 19 patients with CNS AE related to ICI, in which limbic encephalitis was the most frequent diagnosis, occurring in 8 of them. In the clinical picture it predominated altered mental status, memory disturbances, psychiatric complaints and seizures. Interestingly, 3 patients had diencephalic symptoms associated (increased weight, loss of libido and narcolepsy / cataplexy). Brain image showed temporal lobe hyperintensities in $62 \%$ with the rest presenting with no alterations. CSF with inflammatory signs predominated and 7 out of 8 patients were positive for Anti-Ma2 antibodies. Despite immunotherapy, 4 patients died due to the encephalitis and only one achieved full recovery [58]. These findings are in agreement with other cohorts and case reports [59] and pharmacovigilance data with just $30 \%$ of the patients with encephalitis reaching full recovery [8].

Autoimmune encephalitis may be associated with the presence of antibodies, such as antigen $\mathrm{D}(\mathrm{HuD})$, specific antibody to type 2 protein (CASPR2) associated with contactin, glutamic acid decarboxylase (GAD), and specific antibodies to the N-methyl-d-aspartate receptor (NMDA). Therefore, it is necessary to identify whether the patient has a positive antibody for autoimmune or onconeuronal encephalitis [24,56]. It is also worth mentioning that there is an induction of the paraneoplastic neurological syndrome associated with the anti-Ma2 antibody (Ma2PNS), characterized by a specific form of encephalitis with prominent involvement of limbic structures, brainstem and diencephalic, usually in association with testicular or pulmonary disease and brings a poor prognosis to patients [58]. In the retrospective study by Vogrig et al. (2019), which assessed the frequency of anti-Ma2 encephalitis associated with ICI, 4 of the 6 patients died [58].

According to Larkin J et al. (2017), when a patient under ICI presents neurological adverse events (nAE), exclusion diagnosis should be performed, since encephalitis of viral etiology and other diseases that mimic the manifestation of autoimmune encephalitis [16,20,24,55]. As for the treatment, the recommended is the use of high doses of steroids, immunoglobulins or rituximab [15,24,56].

\subsection{Meningoenchepalites}

Vogriev et al. (2020) reported in their retrospective study that immunemediated meningoencephalitis may also be associated with the use of ICI. 4 out of 19 patients with neurological complications associated to ICI had meningoencephalitis diagnosis, presenting with altered mental status (3/4), fever (3/4), anterograde memory disturbances in three (3/4) and headache (1/4). Involvement of the peripheral nervous system has also been reported in 2 patients: one sensorimotor peripheral neuropathy and one sensory neuronopathy. CSF analysis showed inflammatory changes in all cases. Antibodies against glial fibrillary acidic protein (GFAP) were detected in two out of four (50\%) patients in the CSF. Patients' treatments consisted in Nivolumab (2/4), pembrolizumab (1/4), nivolumab and ipilimumab (1/4) [60]. The proposed treatment was withdrawal of ICI and use of bolus corticosteroids in all patients. Finally, in a median follow-up period of 14 months, there was neurological improvement in 2 patients, and one died due to the evolution of the underlying oncological disease [60]

\subsection{Cerebellitis}

Vogrig et al. (2020) reported 4 cases of cerebellitis: 3 of them manifested isolated cerebellar syndrome, while the remaining one also presented cranial nerve involvement (diplopia and vestibulocochlear dysfunction).
Cerebellar disorder was observed in 4 patients (100\%) [60]. MRI was normal in 3 out of 4 patients, while mild cerebellar atrophy was observed in one. Also, one patient had positive anti-Hu antibodies. The proposed treatment was the removal of ICI and boluses of corticosteroids in all patients, and Hu-PNS was associated with IVIG. Two patients recovered completely, one showed remaining symptoms, without disability [60]. Therefore, patients using ICI with evidence of immune-mediated cerebellitis, prompt diagnosis is required, in addition to early treatment of high-dose corticosteroids are essential for a good prognosis, improvement of clinical symptoms and successful treatment, including prevention of hydrocephalus and tonsillar hernia [61].

\subsection{Aseptic meningitis}

In a post marketing study, the prevalence of meningitis related to ICI corresponded to $0.36 \%$ of the AE from ICIs use. Out of 27 patients, 2 died from this complication [8]. Another study found 2 cases of meningitis coexisting with peripheral phenotypes (CIDP and bilateral facial nerve palsy and hearing loss) [14].

When approaching a case of possible meningitis due to ICIs use, the two major differential diagnoses are infectious meningitis and carcinomatous meningitis [62]. Interestedly, in Vogrig et al. study, 4 out of 19 patients with CNS complications had meningoencephalitis associated with an image that could be mistaken for carcinomatous meningitis [60]. The proper evaluation requires CSF analysis and brain MRI after a carefully neurological and systemic examination to rule out any other complication associated. If infection is highly suspicious, proper treatment should be initiated until it can be ruled out. After aseptic meningitis diagnosis confirmations, treatment requires high doses steroids and suspension of the ICIs [62].

\subsection{Posterior reversible encephalopathy syndrome}

Posterior reversible encephalopathy syndrome (PRES) is a rare neurological condition characterized by headache, visual field deficits, focal neurological deficits and seizures [63]. In a study with 11 patients with ICI related CNS adverse events, only 1 had PRES diagnosis [64]. Additionally, PRES have been reported in unusual association with Hashimoto's thyroiditis and stiff person syndrome in an adolescent patient in use of Nivolumab, with good response to immunotherapy [65]. 3.6. Central Nervous Demyelinating Syndromes

Demyelinating syndromes have been related either as relapses in multiple sclerosis (MS) patients [66] or new onset demyelinating syndrome [67]. Isolated myelitis is not a common event in the setting of ICIs treatment ${ }^{6}$. Isolated cases of optic neuritis have been reported as well [68]. These are very rare $\mathrm{AE}$, but they should make part of differential diagnosis of CNS phenotypes in patients in use of ICIs.

\subsection{Paraneoplastic syndromes}

Paraneoplastic neurological syndromes are disorders associated to an immune-mediated response against a subjacent tumor, whether it is benign or malignant. These tumors tend to express onconeural antigens, which are proteins normally present in neuronal cells. Paraneoplastic neurological syndromes may affect any part of the nervous system and frequently occur before the cancer diagnosis [69-71]. However, since ICI increases the immune-response and interferes in self-tolerance, the possibility of paraneoplastic development in face of ICI treatment has emerged.

In this context, Yshii et al. (2016) investigated if anti-CTLA4 therapy could increase the risk of developing paraneoplastic neurological disorders, more specifically paraneoplastic cerebellar degeneration (PCD) 
[70]. PCD occurs due the immune response against intracellular antigens and leads to loss of Purkinje cells in the cerebellum. It is usually related to gynecological (ovarian or breast), testicular or small cell lung cancer [69,70]. Interestedly, 84\% (27/32) of the mices receiving anti-CTLA4 treatment presented cerebellar inflammation, which was not observed in any of the mices not receiving this treatment. Besides that, when Purkinje neurons expressed onconeural antigens, $\mathrm{T}$ cells were recruited and neuronal destruction was observed [70]

Therefore, paraneoplastic neurological disorders must be reminded when a patient develops neurological irAE, especially when symptoms are identical to those in classic paraneoplastic neurological syndrome or when neurological irAE is accompanied by onconeural autoantibodies [69].

A few case series of patients in ICI therapy who developed paraneoplastic neurological syndrome have been reported, including patients with lung adenocarcinoma and Merkel carcinoma in use of pembrolizumab developing sensory neuropathy [71] and small cell lung cancer patients developing encephalitis in use of nivolumab with and without ipilimumab [72]. In some articles, however, although patients developed encephalitis after the use of ICI and had positive autoantibodies in CSF, diagnosis of paraneoplastic neurological disorders have not been made due the atypical clinical presentation [73]

Diagnosis of paraneoplastic neurological syndrome may be challenging. Neural antibody testing is crucial in face of a patient developing neurological irAE after the use of ICI, such as further studies about this association and clinical trials with more detailed clinical descriptions of neurological irAE $[69,71]$.

\section{Conclusion}

In conclusion, $\mathrm{nAE}$ are uncommon but represent a serious complication that can impact patients' prognosis and even be the cause of death in some cases. Neurologists and oncologists should be aware of such complications and be especially alert to the clinical overlap of myocarditis and neuromuscular complications which requires intensive care management and are correlated with high fatality rates.

Ethical statement: The authors are accountable for all aspects of the work in ensuring that questions related to the accuracy or integrity of any part of the work are appropriately investigated and resolved

Conflict of interests: The authors declare that the review article in question has no conflict of interest.

Funding statement: the authors claim to have no financial source in the article.

Acknowledgments: We would like to thank everyone involved in making the article in question, especially Dr. and professor Marcus Vinicius Magno Gonçalves for agreeing to participate and contribute brilliantly to the article.

\section{References}

4. Pardoll DM. The blockade of immune checkpoints in cancer immunotherapy. Nat Rev Cancer. 2012. doi:10.1038/nrc3239

5. El Osta B, Hu F, Sadek R, Chintalapally R, Tang SC. Not all immune-checkpoint inhibitors are created equal: Meta-analysis and systematic review of immune-related adverse events in cancer trials. Crit Rev Oncol Hematol. 2017.

6. Shimozaki K, Sukawa Y, Beppu N, et al. Multiple immunerelated adverse events and anti-tumor efficacy: Real-world data on various solid tumors. Cancer Manag Res. 2020;12:4585-
4593.

7. Man J, Ritchie G, Links M, Lord S, Lee CK. Treatment-related toxicities of immune checkpoint inhibitors in advanced cancers: A meta-analysis. Asia Pac J Clin Oncol. 2018.

8. Abdelhafeez AAM, Shohdy KS, Ibrahim W. Safety of Combination Immune Checkpoint Inhibitors Compared to Monotherapy; A Systematic Review and Meta-Analysis. Cancer Invest. 2020;38(3):150-157.

9. Cuzzubbo S, Javeri F, Tissier M, et al. Neurological adverse events associated with immune checkpoint inhibitors: Review of the literature. Eur J Cancer. 2017.

10. Mancone S, Lycan T, Ahmed T, et al. Severe neurologic complications of immune checkpoint inhibitors: a single-center review. J Neurol. 2018. doi:10.1007/s00415-018-8890-z

11. Sato K, Mano T, Iwata A, Toda T. Neurological and related adverse events in immune checkpoint inhibitors: a pharmacovigilance study from the Japanese Adverse Drug Event Report database. J Neurooncol. 2019. doi:10.1007/s11060-019-03273-1

12. Wang DY, Salem J-E, Cohen J V., et al. Fatal Toxic Effects Associated With Immune Checkpoint Inhibitors. JAMA Oncol. 2018. doi:10.1001/jamaoncol.2018.3923

13. Moreira A, Loquai C, Pföhler C, et al. Myositis and neuromuscular side-effects induced by immune checkpoint inhibitors. Eur J Cancer. 2019. doi:10.1016/j.ejca.2018.09.033

14. Salem JE, Manouchehri A, Moey M, et al. Cardiovascular toxicities associated with immune checkpoint inhibitors: an observational, retrospective, pharmacovigilance study. Lancet Oncol. 2018. doi:10.1016/S1470-2045(18)30608-9

15. Suzuki S, Ishikawa N, Konoeda F, et al. Nivolumab-related myasthenia gravis with myositis and myocarditis in Japan. Neurology. 2017. doi:10.1212/WNL.0000000000004359

16. Weber JS, Kähler KC, Hauschild A. Management of immunerelated adverse events and kinetics of response with ipilimumab. J Clin Oncol. 2012 . doi:10.1200/JCO.2012.41.6750

17. Dubey D, David WS, Amato AA, et al. Varied phenotypes and management of immune checkpoint inhibitor-Associated neuropathies. Neurology. 2019. doi:10.1212/WNL.0000000000008091

18. Hottinger AF. Neurologic complications of immune checkpoint inhibitors. Curr Opin Neurol. 2016. doi:10.1097/WCO.0000000000000391

19. Astaras C, de Micheli R, Moura B, Hundsberger T, Hottinger AF. Neurological Adverse Events Associated with Immune Checkpoint Inhibitors: Diagnosis and Management. Curr Neurol Neurosci Rep. 2018. doi:10.1007/s11910-018-0810-1

20. Postow MA, Sidlow R, Hellmann MD. Immune-related adverse events associated with immune checkpoint blockade. N Engl J Med. 2018. doi:10.1056/NEJMra1703481

21. Fellner A, Makranz C, Lotem M, et al. Neurologic complications of immune checkpoint inhibitors. J Neurooncol. 2018. doi:10.1007/s11060-018-2752-5

22. Yshii LM, Hohlfeld R, Liblau RS. Inflammatory CNS disease caused by immune checkpoint inhibitors: Status and perspectives. Nat Rev Neurol. 2017. 
doi:10.1038/nrneurol.2017.144

23. Larkin J, Chmielowski B, Lao CD, et al. Neurologic Serious Adverse Events Associated with Nivolumab Plus Ipilimumab or Nivolumab Alone in Advanced Melanoma, Including a Case Series of Encephalitis. Oncologist. 2017. doi:10.1634/theoncologist.2016-0487

24. Voskens CJ, Goldinger SM, Loquai C, et al. The Price of Tumor Control: An Analysis of Rare Side Effects of Anti-CTLA-4 Therapy in Metastatic Melanoma from the Ipilimumab Network. PLoS One. 2013. doi:10.1371/journal.pone.0053745

25. Bompaire F, Mateus C, Taillia H, et al. Severe meningoradiculo-nevritis associated with ipilimumab. Invest New Drugs. 2012. doi:10.1007/s10637-011-9787-1

26. Manousakis G, Koch J, Sommerville RB, et al. Multifocal radiculoneuropathy during ipilimumab treatment of melanoma. Muscle and Nerve. 2013. doi:10.1002/mus.23830

27. Santomasso BD. Anticancer Drugs and the Nervous System. Continuum (Minneap Minn). 2020. doi:10.1212/CON.0000000000000873

28. Alhammad RM, Dronca RS, Kottschade LA, et al. Brachial Plexus Neuritis Associated With Anti-Programmed Cell Death-1 Antibodies: Report of 2 Cases. Mayo Clin Proc Innov Qual Outcomes. 2017. doi:10.1016/j.mayocpiqo.2017.07.004

29. Haugh AM, Probasco JC, Johnson DB. Neurologic complications of immune checkpoint inhibitors. Expert Opin Drug Saf. 2020. doi:10.1080/14740338.2020.1738382

30. Malek E, Salameh J. Guillain-Barre Syndrome. Semin Neurol. 2019. doi:10.1055/s-0039-1693005

31. Gu Y, Menzies AM, Long G V., Fernando SL, Herkes G. Immune mediated neuropathy following checkpoint immunotherapy. J Clin Neurosci. 2017. doi:10.1016/j.jocn.2017.07.014

32. Wilgenhof S, Neyns B. Anti-CTLA-4 antibody-induced Guillain-Barré syndrome in a melanoma patient. Ann Oncol. 2011. doi:10.1093/annonc/mdr028

33. Gravbrot N, Scherer K, Sundararajan S. Safe Transition to Pembrolizumab following Ipilimumab-Induced Guillain-Barré Syndrome: A Case Report and Review of the Literature. Case Rep Oncol Med. 2019. doi:10.1155/2019/5490707

34. Supakornnumporn S, Katirji B. Guillain-Barré syndrome triggered by immune checkpoint inhibitors: A case report and literature review. J Clin Neuromuscul Dis. 2017. doi:10.1097/CND.0000000000000193

35. Möhn N, Beutel G, Gutzmer R, Ivanyi P, Satzger I, Skripu T. Neurological Immune Related Adverse Events Associated with Nivolumab, Ipilimumab, and Pembrolizumab TherapyReview of the Literature and Future Outlook. J Clin Med. 2019. doi:10.3390/jcm8111777

36. McNeill CJ, Fehmi J, Gladwin J, Price C. A rare case of Miller Fisher variant of Guillain-Barr é Syndrome (GBS) induced by a checkpoint inhibitor. BMJ Case Rep. 2019. doi:10.1136/bcr2019-229443

37. Kolb NA, Trevino CR, Waheed W, et al. Neuromuscular complications of immune checkpoint inhibitor therapy. Muscle and Nerve. 2018. doi:10.1002/mus.26070

38. Rajabally YA, Attarian S. Chronic inflammatory demyelinating polyneuropathy and malignancy: A systematic review. Muscle and Nerve. 2018. doi:10.1002/mus.26028

39. Hughes RAC, Swan A V., van Doorn PA. Intravenous immunoglobulin for Guillain-Barré syndrome. Cochrane Database Syst Rev. 2014. doi:10.1002/14651858.CD002063.pub6

40. Tanaka R, Maruyama H, Tomidokoro Y, et al. Nivolumabinduced chronic inflammatory demyelinating polyradiculoneuropathy mimicking rapid-onset Guillain-Barré syndrome: A case report. Jpn J Clin Oncol. 2016. doi:10.1093/jjco/hyw090

41. Selva-O'Callaghan A, Pinal-Fernandez I, Trallero-Araguás E, Milisenda JC, Grau-Junyent JM, Mammen AL. Classification and management of adult inflammatory myopathies. Lancet Neurol. 2018. doi:10.1016/S1474-4422(18)30254-0

42. Milone M. Diagnosis and Management of Immune-Mediated Myopathies. Mayo Clin Proc. 2017. doi:10.1016/j.mayocp.2016.12.025

43. Eggermont AMM, Blank CU, Mandala M, et al. Adjuvant pembrolizumab versus placebo in resected stage III melanoma. N Engl J Med. 2018. doi:10.1056/NEJMoa1802357

44. Touat M, Maisonobe T, Knauss S, et al. Immune checkpoint inhibitor-related myositis and myocarditis in patients with cancer. Neurology. 2018. doi:10.1212/WNL.0000000000006124

45. Bruna J, Argyriou AA, Anastopoulou GG, et al. Incidence and characteristics of neurotoxicity in immune checkpoint inhibitors with focus on neuromuscular events: Experience beyond the clinical trials. J Peripher Nerv Syst. 2020;25(2):171177. doi:10.1111/jns.12371

46. Kang KH, Grubb W, Sawlani K, et al. Immune checkpointmediated myositis and myasthenia gravis: A case report and review of evaluation and management. Am J Otolaryngol Head Neck Med Surg. 2018. doi:10.1016/j.amjoto.2018.06.003

47. Benfaremo D, Manfredi L, Luchetti MM, Gabrielli A. Musculoskeletal and Rheumatic Diseases Induced by Immune Checkpoint Inhibitors: A Review of the Literature. Curr Drug Saf. 2018. doi:10.2174/1574886313666180508122332

48. Touat M, Talmasov D, Ricard D, Psimaras D. Neurological toxicities associated with immune-checkpoint inhibitors. Curr Opin Neurol. 2017. doi:10.1097/WCO.0000000000000503

49. Liao B, Shroff S, Kamiya-Matsuoka C, Tummala S. Atypical neurological complications of ipilimumab therapy in patients with metastatic melanoma. Neuro Oncol. 2014. doi:10.1093/neuonc/nou001

50. Hunter G, Voll C, Robinson CA. Autoimmune inflammatory myopathy after treatment with ipilimumab. Can J Neurol Sci. 2009. doi:10.1017/S0317167100007939

51. Gonzalez NL, Puwanant A, Lu A, Marks SM, Živković SA. Myasthenia triggered by immune checkpoint inhibitors: New case and literature review. Neuromuscul Disord. 2017. doi:10.1016/j.nmd.2017.01.002

52. Liewluck T, Kao JC, Mauermann ML. PD-1 Inhibitorassociated Myopathies: Emerging Immune-mediated Myopathies. J Immunother. 2018. doi:10.1097/CJI.0000000000000196 
53. Psimaras D, Velasco R, Birzu C, et al. Immune checkpoint inhibitors-induced neuromuscular toxicity: From pathogenesis to treatment. J Peripher Nerv Syst. 2019. doi:10.1111/jns. 12339

54. Safa H, Johnson DH, Trinh VA, et al. Immune checkpoint inhibitor related myasthenia gravis: Single center experience and systematic review of the literature. J Immunother Cancer. 2019. doi:10.1186/s40425-019-0774-y

55. Psimaras D. Neuromuscular complications of immune checkpoint inhibitors. Press Medicale. 2018. doi:10.1016/j.lpm.2018.10.009

56. Grob D, Brunner N, Namba T, Pagala M. Lifetime course of myasthenia gravis. Muscle and Nerve. 2008. doi:10.1002/mus.20950

57. Wendell LC, Levine JM. Myasthenic Crisis. The Neurohospitalist. 2011. doi:10.1177/1941875210382918

58. Perrinjaquet C, Desbaillets N, Hottinger AF. Neurotoxicity associated with cancer immunotherapy: Immune checkpoint inhibitors and chimeric antigen receptor T-cell therapy. Curr Opin Neurol. 2019. doi:10.1097/WCO.0000000000000686

59. Dalakas MC. Neurological complications of immune checkpoint inhibitors: what happens when you 'take the brakes off' the immune system. Ther Adv Neurol Disord. 2018. doi:10.1177/1756286418799864

60. Salam S, Lavin T, Turan A. Limbic encephalitis following immunotherapy against metastatic malignant melanoma. BMJ Case Rep. 2016. doi:10.1136/bcr-2016-215012

61. Vogrig A, Fouret M, Joubert B, et al. Increased frequency of anti-Ma2 encephalitis associated with immune checkpoint inhibitors. Neurol Neuroimmunol NeuroInflammation. 2019. doi:10.1212/NXI.0000000000000604

62. Galmiche S, Lheure C, Kramkimel N, et al. Encephalitis induced by immune checkpoint inhibitors in metastatic melanoma: a monocentric retrospective study. J Eur Acad Dermatology Venereol. 2019. doi:10.1111/jdv.15756

63. Vogrig A, Vogrig A, Vogrig A, et al. Central nervous system complications associated with immune checkpoint inhibitors. J Neurol Neurosurg Psychiatry. 2020. doi:10.1136/jnnp-2020323055

64. Zurko J, Mehta A. Association of Immune-Mediated Cerebellitis With Immune Checkpoint Inhibitor Therapy. Mayo Clin Proc Innov Qual Outcomes. 2018. doi:10.1016/j.mayocpiqo.2017.12.001
65. Reynolds KL, Guidon AC. Diagnosis and Management of Immune Checkpoint Inhibitor-Associated Neurologic Toxicity: Illustrative Case and Review of the Literature. Oncologist. 2019. doi:10.1634/theoncologist.2018-0359

66. Kim D. Posterior reversible encephalopathy syndrome induced by nivolumab immunotherapy for non-small-cell lung cancer. Clin Case Reports. 2019. doi:10.1002/ccr3.2122

67. Dubey D, David WS, Reynolds KL, et al. Severe Neurological Toxicity of Immune Checkpoint Inhibitors: Growing Spectrum. Ann Neurol. 2020. doi:10.1002/ana.25708

68. Tchapyjnikov D, Borst AJ. Immune-related Neurological Symptoms in an Adolescent Patient Receiving the Checkpoint Inhibitor Nivolumab. J Immunother. 2017. doi:10.1097/CJI.0000000000000177

69. Garcia CR, Jayswal R, Adams V, Anthony LB, Villano JL. Multiple sclerosis outcomes after cancer immunotherapy. Clin Transl Oncol. 2019. doi:10.1007/s12094-019-02060-8

70. Romeo MAL, Garassino MC, Moiola L, et al. Multiple sclerosis associated with pembrolizumab in a patient with non-small cell lung cancer. J Neurol. 2019. doi:10.1007/s00415-019-09562-Z

71. Wilson MA, Guld K, Galetta S, et al. Acute visual loss after ipilimumab treatment for metastatic melanoma. J Immunother Cancer. 2016. doi:10.1186/s40425-016-0170-9

72. Graus F, Dalmau J. Paraneoplastic neurological syndromes in the era of immune-checkpoint inhibitors. Nat Rev Clin Oncol. 2019. doi:10.1038/s41571-019-0194-4

73. Yshii LM, Gebauer CM, Pignolet B, et al. CTLA4 blockade elicits paraneoplastic neurological disease in a mouse model. Brain. 2016;139(11):2923-2934. doi:10.1093/brain/aww225

74. Gill AJ, Perez MA, Perrone CM, Bae CJ, Pruitt AA, Lancaster E. A case series of PD-1 inhibitor-associated paraneoplastic neurologic syndromes. J Neuroimmunol. 2019;334(April):576980. doi:10.1016/j.jneuroim.2019.576980

75. Antonia SJ, López-Martin JA, Bendell J, et al. Nivolumab alone and nivolumab plus ipilimumab in recurrent small-cell lung cancer (CheckMate 032): a multicentre, open-label, phase 1/2 trial. Lancet Oncol. 2016;17(7):883-895. doi:10.1016/S14702045(16)30098-5

76. Williams TJ, Benavides DR, Patrice KA, et al. Association of autoimmune encephalitis with combined immune checkpoint inhibitor treatment for metastatic cancer. JAMA Neurol. 2016;73(8):928-933. doi:10.1001/jamaneurol.2016.1399 
cC) (†) This work is licensed under Creative Commons Attribution 4.0 License

To Submit Your Article Click Here: Submit Manuscript

DOI:10.31579/2578-8868/177
Ready to submit your research? Choose Auctores and benefit from:

* fast, convenient online submission

* rigorous peer review by experienced research in your field

* rapid publication on acceptance

* authors retain copyrights

* unique DOI for all articles

* immediate, unrestricted online access

At Auctores, research is always in progress.

Learn more www.auctoresonline.org/journals/neuroscience-andneurological-surgery 